\title{
Silencing of the transcriptional factor ZEB1 alters the steroidogenic pathway, and increases the concentration of testosterone and DHT in DU145 cells
}

\author{
D. HERRERA ${ }^{1}$, O. ORELLANA-SERRADELL ${ }^{1}$, P. VILLAR ${ }^{1}$, M.J. TORRES ${ }^{1}$, \\ R. PACIUCCI ${ }^{2}$, E.A. CASTELLÓN ${ }^{1}$ and H.R. CONTRERAS ${ }^{1}$ \\ ${ }^{1}$ Department of Basic and Clinic Oncology, Faculty of Medicine, University of Chile, 8380453 Santiago, Chile; \\ ${ }^{2}$ Biomedical Research Group of Urology, Vall d'Hebron Research Institute, \\ Universitat Autònoma de Barcelona, 08035 Barcelona, Spain
}

Received July 26, 2018; Accepted November 8, 2018

DOI: $10.3892 /$ or.2018.6885

\begin{abstract}
Prostate cancer (PCa) is the second most common type of male malignancy worldwide. The transcription factor zinc fingerE-box binding homeobox 1 (ZEB1) is associated with epithelial-mesenchymal transition and is also involved in regulation of androgen receptor (AR) expression, the main ligands of which are testosterone and dihydrotestosterone (DHT). These androgens are synthesized through the steroidogenic pathway within the prostate, and their synthesis is altered in PCa. The present study aimed to determine the ZEB1-induced alterations in androgen synthesis and AR expression in the DU145 PCa cell line. Reverse transcription-quantitative polymerase chain reaction, western blotting and immunocytochemistry were used to determine the mRNA and protein expression levels, and cellular localization of steroidogenic pathway enzymes in the DU145 cell line in response to ZEB1 silencing. Furthermore, the concentrations of testosterone and DHT were detected in cell culture medium using ELISA. ZEB1-silenced cells exhibited an increase in testosterone and DHT production, an increase in AR expression and an alteration in the steroidogenic pathway. In particular, steroidogenic acute regulatory protein and $5 \alpha$-reductase 2 expression levels were decreased, whereas cytochrome $\mathrm{P} 450$ family 17 subfamily A member $1,5 \alpha$-reductase 1 , aldo-keto reductase family 1 member D1 and aldo-keto reductase family 1 member $\mathrm{C} 2$ expression levels were increased. In conclusion, the present study provided novel information regarding the regulation of intratumoral androgen production in $\mathrm{PCa}$, which is relevant for the progression of the disease to a castration-resistant form.
\end{abstract}

Correspondence to: Dr H.R. Contreras, Department of Basic and Clinic Oncology, Faculty of Medicine, University of Chile, Avenida Independencia 1027, 8380453 Santiago, Chile

E-mail: hcontrer@med.uchile.cl

Key words: steroidogenic pathway, ZEB1, AR, androgens, prostate cancer

\section{Introduction}

Prostate cancer (PCa) is the second most common type of male malignancy worldwide and $\sim 1.3$ million new cases of $\mathrm{PCa}$ are predicted to occur in 2018. Furthermore, $\mathrm{PCa}$ is the fifth leading cause of cancer-associated mortality in men, and $\sim 359,000$ cases of PCa-associated mortality are predicted to occur worldwide in 2018 (1).

Androgens have an important role in the development and growth of the normal prostate gland, as well as in the proliferation of PCa cells $(2,3)$. It has been reported that activation of the androgen receptor (AR) promotes the proliferation of $\mathrm{PCa}$ (3). Therefore, androgen deprivation therapy (ADT) is considered the gold standard for the treatment of PCa recurrence and metastasis. However, despite a good initial response to ADT, the majority of patients progress to aggressive castration-resistant PCa (CRPC) within 2-3 years $(2,4)$. Various mechanisms underlying resistance to ADT have been identified, including AR hypersensitivity, mutations, amplification and splicing variants, in addition to intratumoral steroidogenesis $(3,4)$. Regarding intratumoral steroidogenesis, distinct patterns of dysregulated expression of enzymes involved in androgen synthesis and metabolism have been reported, indicating that tumor cells from patients with CRPC exhibit increased expression of the steroidogenic enzymes hydroxysteroid dehydrogenase (HSD) $3 \beta 1$, HSD3 $\beta 2$, HSD17 $\beta 3$, aldo-keto reductase family 1 member C3 (AKR1C3) and 5 $\alpha$-reductase $1(5,6)$.

Notably, ADT may induce epithelial-mesenchymal transition (EMT); activation of this transdifferentiation program may increase tumor malignancy (7). A previous study demonstrated the importance of zinc finger E-box binding homeobox 1 (ZEB1), which is a canonical transcription factor of EMT, since it is not only a key EMT factor, but also an AR regulator (8). In the PC3 cell line, a negative loop of regulation has been identified between ZEB1 and the AR, resulting in decreased levels of AR in response to high ZEB1 expression and vice versa. Furthermore, the AR is a direct regulator of the ZEB1 gene, since it binds to two androgen response elements located 1,000 base pairs near the site of transcriptional initiation (8). However, to the best of our knowledge, the alterations 
occurring in androgen synthesis in relation to ZEB1 have not yet been established. Therefore, it may be hypothesized that ZEB1 alters androgen synthesis capacity in PCa.

The present study aimed to determine the alterations in androgen synthesis and AR expression induced by ZEB1 knockdown in the PCa cell line DU145. The results indicated that ZEB1 silencing significantly altered the expression of the cholesterol transporter steroidogenic acute regulatory protein (StAR), and enzymes involved in the synthesis and degradation of androgens, including cytochrome $\mathrm{P} 450$ family 17 subfamily A member 1 (CYP17A1), 5 $\alpha$-reductase 1, $5 \alpha$-reductase 2 , aldo-keto reductase family 1 member D1 (AKR1D1) and aldo-keto reductase family 1 member C2 (AKR1C2), thus resulting in an increase in testosterone and dihydrotestosterone (DHT) concentration in the cell culture medium. These findings may provide novel information regarding the regulation of androgen synthesis in PCa.

\section{Materials and methods}

Cell culture and transduction. The PCa cell line DU145 [cat. no. American Type Culture Collection (ATCC) HTB-81] was obtained from the ATCC (Manassas, VA, USA). ZEB1-knockdown cells (DU145 SH) and control cells (DU145 SCR) were produced in our laboratory according to protocols described in our previous study (9). Briefly, 5.5x $10^{4}$ cells were transduced with $1 \mu \mathrm{g}$ lentiviral vector containing a short hairpin (sh)RNA against ZEB1 [pLenti-6-shRNA (h ZEB1)-Rsv (RFP-Puro)] (GenTarget, Inc., San Diego, CA, USA). A shRNA against a random sequence was used as a control [pLenti-U6-shRNA (neg-control)-Rsv (RFP-Puro)] (GenTarget, Inc.). Subsequently, cells were selected with $1.5 \mu \mathrm{g} / \mathrm{ml}$ puromycin and effective silencing was verified using fluorescence microscopy, reverse transcription-quantitative polymerase chain reaction (RT-qPCR) and western blotting (9). These cell lines were cultured in RPMI-Phenol Red-free culture medium (Gibco; Thermo Fisher Scientific, Inc., Waltham, MA, USA) supplemented with $10 \%$ fetal bovine serum (Corning Incorporated, Corning, NY, USA) and were incubated at $37^{\circ} \mathrm{C}$ in an atmosphere containing $5 \% \mathrm{CO}_{2}$.

$R T-q P C R$. The cells were harvested and lysed using TRIzol ${ }^{\circledR}$ reagent (cat. no. 15596-026; Invitrogen; Thermo Fisher Scientific, Inc.). RNA extraction was conducted according to standard procedures, and RNA was quantified using a Synergy HT Multi-Detection Microplate Reader (Biotek Instruments, Inc., Winooski, VT, USA). Subsequently, 100 ng total RNA was reverse transcribed into cDNA using the Affinityscript QPCR cDNA Synthesis commercial kit (Agilent Technologies, Inc., Santa Clara, CA, USA). The templates were amplified in one cycle of $5 \mathrm{~min}$ at $25^{\circ} \mathrm{C}, 45 \mathrm{~min}$ at $42^{\circ} \mathrm{C}$ and $5 \mathrm{~min}$ at $95^{\circ} \mathrm{C}$. qPCR was then performed using the SYBR green QPCR Master Mix kit (Agilent Technologies, Inc.) in an Aria Mix Real-Time PCR system (Agilent Technologies, Inc.), according to the manufacturer's protocol. The templates were amplified as follows: One cycle at $95^{\circ} \mathrm{C}$ for $10 \mathrm{~min}$, followed by 40 cycles at $95^{\circ} \mathrm{C}$ for $15 \mathrm{~min}, 60^{\circ} \mathrm{C}$ for $15 \mathrm{~min}$ and $72^{\circ} \mathrm{C}$ for $15 \mathrm{~min}$, and one cycle at $95^{\circ} \mathrm{C}$ for $15 \mathrm{~min}, 65^{\circ} \mathrm{C}$ for $15 \mathrm{~min}$ and $95^{\circ} \mathrm{C}$ for $15 \mathrm{~min}$. Finally, data were analyzed using the AriaMX 1.0 program (Agilent Technologies, Inc.). The primers used are shown in Table I. The quantification of gene expression was performed in triplicate using the $2^{-\Delta \Delta C q}$ method and expression levels were normalized to those of the housekeeping gene, pumilio RNA binding family member 1 (10).

Western blotting. For protein extraction, cells were harvested and were treated with radioimmunoprecipitation assay lysis buffer mixed with protease inhibitor (Roche Diagnostics, Basel, Switzerland). The obtained homogenate was centrifuged at 26,500 x g (Beckman Coulter Allegra ${ }^{\circledR} 21 \mathrm{R}$; Beckman Coulter, Inc., Brea, CA, USA) for $15 \mathrm{~min}$ at $4^{\circ} \mathrm{C}$. Finally, the supernatant was quantified using the Bradford method (Bio-Rad Laboratories, Inc., Hercules, CA, USA), according to the manufacturer's protocol. Subsequently, $50 \mu \mathrm{g}$ protein under reducing conditions was separated by $10 \%$ acrylamide gel electrophoresis and the proteins were electrotransferred onto nitrocellulose membranes (Bio-Rad Laboratories, Inc.) at $50 \mathrm{~mA}$ and $4^{\circ} \mathrm{C}$ overnight. Afterwards, the membranes were blocked with $5 \%$ milk solution in TBS-Tween $0.1 \%$ (TBST) for $2 \mathrm{~h}$ at room temperature, washed with TBST, and incubated overnight at $4^{\circ} \mathrm{C}$ with the corresponding primary antibodies (Table II). The membranes were then washed and incubated with the horseradish peroxidase-conjugated respective secondary antibodies for $1 \mathrm{~h}$ at room temperature (Table II). Finally, the membranes were developed by chemiluminescence using an automatic system (Fusion FX5-XT; Vilber Lourmat Sté, Collégien, France). Semi-quantification of protein expression levels was conducted using ImageJ 1.52f software (National Institutes of Health, Bethesda, MD, USA), with $\beta$-actin used as a loading control.

Determination of testosterone and DHT levels in cell culture medium. A total of $4 \times 10^{5}$ DU145 SH and DU145 SCR cells/plate were seeded into $100 \mathrm{~mm}$ plates containing $12 \mathrm{ml}$ RPMI-Phenol red-free medium, and were cultured for 3 days. Subsequently, cells were counted using a Neubauer chamber. For steroid extraction, $300 \mu \mathrm{l}$ culture media was removed and diethylether was added in a 5:1 ratio (solvent/sample). The samples were then vortexed for 2 min and incubated at $4^{\circ} \mathrm{C}$ for $5 \mathrm{~min}$, in order to allow correct separation of the phases. Subsequently, the organic phase was removed and evaporated in SpeedVac (Savant ${ }^{\mathrm{TM}}$ SC110; Thermo Fisher Scientific, Inc.). Finally, the samples were reconstituted in $500 \mu 110 \%$ ethanol or methanol, in order to measure the levels of testosterone or DHT respectively, using an ELISA kit for testosterone serum detection (cat. no. 11-TESHU-E01; Alpco, Salem, MA, USA) and an ELISA kit for DHT serum detection (cat. no. 11-DHTHU-E01; Alpco), with a modification in the construction of the calibration curve. Briefly, since the samples were resuspended in solution with $10 \%$ ethanol or methanol, a novel calibration curve was constructed in these matrices using synthetic testosterone (Sigma-Aldrich; Merck KGaA) and synthetic DHT (Sigma-11-DHTHU-E01) at the same concentrations indicated in the kit calibration curves. To validate the method, a control sample of known concentration was measured and the minimum detectable dose was calculated. Afterwards, the measurement was performed according to the manufacturer protocols. Finally, the results were corrected to determine the concentration obtained for $10^{5}$ cells. 
Table I. Sequences of primers used for reverse transcription-quantitative polymerase chain reaction.

\begin{tabular}{llc}
\hline Marker & \multicolumn{1}{c}{ Forward primer } & \multicolumn{1}{c}{ Reverse primer } \\
\hline StAR & 5'-TCCTTAGCAACCAAGAGGGC-3' & 5'-TGACATTGGGGTTCCACTCC-3' \\
CYP17A1 & 5'-GCCCCATCTATTCGGTTCGT-3' & 5'-CAGAGTCAGCGAAGGCGATA-3' \\
$5 \alpha$-reductase 1 & 5'-CATGTTCCTCGTCCACTACGG-3' & 5'-CCAACAGTGGCATAGGCTTTC-3' \\
$5 \alpha-$-reductase 2 & 5'-TTCCTTCGCGGTGCCC-3' & 5'-CCATTTCCAGTGCAGAAGGC-3' \\
AKR1D1 & 5'-TGGTCACTTCATGCCTGTCC-3' & 5'-CAATATGGCGGAAGCCAGC-3' \\
AKR1C2 & 5'-ACCATTGGAATGACATACTGCATC-3' & 5'-TGTGAGAGGAGGGACAGAGG-3' \\
AR & 5'-TTGTGTCAAAAGCGAAATGG-3' & 5'-AGTCAATGGGCAAAACATGG-3' \\
PUM1 & 5'-CGGTCGTCCTGAGGATAAAA-3' & 5'-CGTACGTGAGGCGTGAGTAA-3'
\end{tabular}

AKR1C2, aldo-keto reductase family 1 member C2; AKR1D1, aldo-keto reductase family 1 member D1; AR, androgen receptor; CYP17A1, cytochrome P450 family 17 subfamily member; PUM1, pumilio RNA binding family member 1; StAR, steroidogenic acute regulatory protein.

Table II. Antibodies used for western blotting.

A, Primary antibodies

\begin{tabular}{|c|c|c|c|}
\hline Antibody name & Catalogue number & Type & Dilution \\
\hline StAR & PA5-21687; Thermo Fisher Scientific, Inc., Waltham, MA, USA & Rabbit & $1: 20$ \\
\hline CYP17A1 & ABC392; EMD Millipore, Billerica, MA, USA & Rabbit & $1: 200$ \\
\hline $5 \alpha$-reductase 1 & ab110123; Abcam, Cambridge, UK & Goat & $1: 500$ \\
\hline $5 \alpha$-reductase 2 & ab27469; Abcam & Goat & $1: 500$ \\
\hline AKR1D1 & PA5-28963; Thermo Fisher Scientific, Inc. & Rabbit & $1: 1,000$ \\
\hline $\mathrm{AKR} 1 \mathrm{C} 2$ & PA5-36572; Thermo Fisher Scientific, Inc. & Rabbit & $1: 500$ \\
\hline AR & ab9474; Abcam & Mouse & $1: 200$ \\
\hline$\beta$-actin & 69100; MP Biomedicals, LLC, Santa Ana, CA, USA & Mouse & $1: 5,000$ \\
\hline
\end{tabular}

B, Secondary antibodies

\begin{tabular}{|c|c|c|c|}
\hline Antibody name & Catalogue number & Type & Dilution \\
\hline Anti-mouse & $\begin{array}{l}\text { 115-035-003; Jackson ImmunoResearch Laboratories, Inc., } \\
\text { West Grove, PA, USA. }\end{array}$ & Goat & $1: 10,000$ \\
\hline Anti-rabbit & 111-035-003; Jackson ImmunoResearch Laboratories, Inc. & Goat & $1: 10,000$ \\
\hline Anti-goat & 305-035-045; Jackson ImmunoResearch Laboratories, Inc. & Rabbit & $1: 10,000$ \\
\hline
\end{tabular}

AKR1C2, aldo-keto reductase family 1 member C2; AKR1D1, aldo-keto reductase family 1 member D1; AR, androgen receptor; CYP17A1, cytochrome P450 family 17 subfamily A member; StAR, steroidogenic acute regulatory protein.

Immunocytochemistry. The DU145 SH and DU145 SCR cells were seeded on coverslips at $60 \%$ confluence and were fixed with $\mathrm{PBS}, 3 \%$ paraformaldehyde and $2 \%$ sucrose for $30 \mathrm{~min}$ at room temperature. Subsequently, cells were washed with PBS-Glycine for $15 \mathrm{~min}$, permeabilized for $10 \mathrm{~min}$ at room temperature, with $0.1 \%$ Triton X-100 (Sigma-Aldrich; Merck KGaA) and washed with PBS-Glycine. Cells were then blocked in a humidified chamber using PBS-Glycine and $1 \%$ bovine serum albumin (Sigma-Aldrich; Merck KGaA) for $10 \mathrm{~min}$ and were incubated with the necessary primary antibodies diluted in blocking solution. The primary antibodies used are shown in
Table III. Subsequently, cells were incubated in the dark with the corresponding Alexa Fluor ${ }^{\circledR} 488$-conjugated secondary antibodies (Table III). Both incubations were performed for $1 \mathrm{~h}$ at $37^{\circ} \mathrm{C}$. In addition, cells were incubated in the dark with DAPI (1:10,000; Santa Cruz Biotechnology, Inc., Dallas, TX, USA) for nuclear staining. Finally, coverslips were washed and mounted with Fluorescence Mounting Medium (Dako; Agilent Technologies, Inc.) for subsequent observation with a Spinning Disk microscope (Olympus IX81; Olympus Corporation, Tokyo, Japan) at x40 magnification. Each of the markers was analyzed in triplicate for DU145 SH and DU145 SCR cells. 
Table III. Antibodies used for immunocytochemistry.

A, Primary antibodies

\begin{tabular}{|c|c|c|c|}
\hline Antibody name & Catalogue number & Type & Dilution \\
\hline StAR & PA5-21687; Thermo Fisher Scientific, Inc., Waltham, MA, USA & Rabbit & $1: 100$ \\
\hline CYP17A1 & ABC392; EMD Millipore, Billerica, MA, USA & Rabbit & $1: 100$ \\
\hline $5 \alpha$-reductase 1 & ab110123; Abcam, Cambridge, UK & Goat & $1: 200$ \\
\hline $5 \alpha$-reductase 2 & ab27469; Abcam & Goat & $1: 200$ \\
\hline AKR1D1 & PA5-28963; Thermo Fisher Scientific, Inc. & Rabbit & $1: 200$ \\
\hline $\mathrm{AKR} 1 \mathrm{C} 2$ & PA5-36572; Thermo Fisher Scientific, Inc. & Rabbit & $1: 200$ \\
\hline AR & ab9474; Abcam & Mouse & $1: 100$ \\
\hline
\end{tabular}

B, Secondary antibodies

\begin{tabular}{|c|c|c|c|}
\hline Antibody name & Catalogue number & Type & Dilution \\
\hline Anti-mouse & A11001; Thermo Fisher Scientific, Inc. & Goat & $1 / 200$ \\
\hline Anti-rabbit & A11008; Thermo Fisher Scientific, Inc. & Goat & $1 / 200$ \\
\hline Anti-goat & ab150141; Abcam & Rabbit & $1 / 200$ \\
\hline
\end{tabular}

AKR1C2, aldo-keto reductase family 1 member C2; AKR1D1, aldo-keto reductase family 1 member D1; AR, androgen receptor; CYP17A1, cytochrome P450 family 17 subfamily A member; StAR, steroidogenic acute regulatory protein.
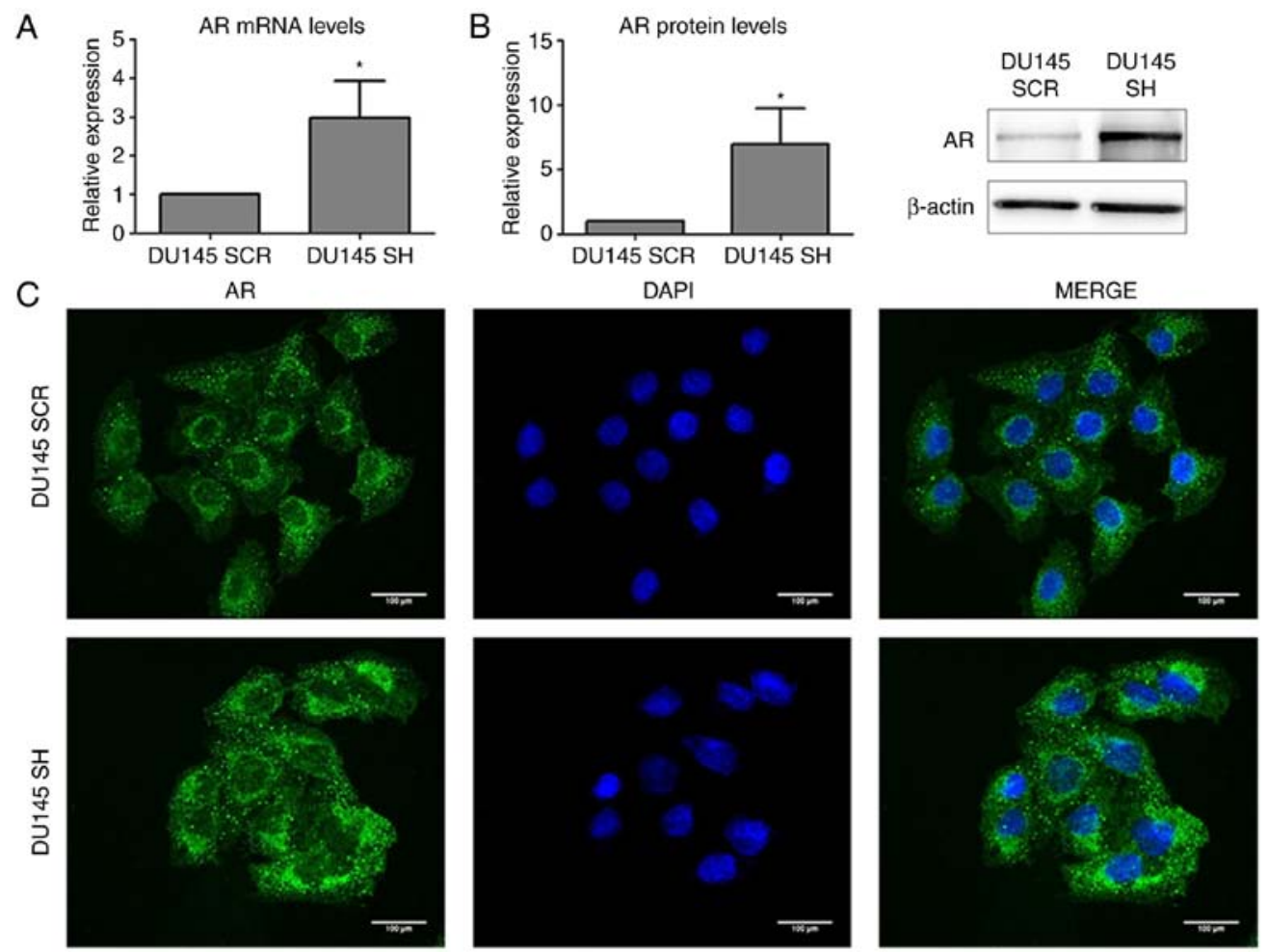

Figure 1. Expression of AR in DU145 SH and DU145 SCR cell lines. (A) AR mRNA expression levels were determined by reverse transcription-quantitative polymerase chain reaction. (B) AR protein expression levels were determined by western blotting. (C) Cellular localization of AR was determined by immunocytochemistry. magnification, $\mathrm{x} 40$. Statistically significant differences were determined using the Mann-Whitney $\mathrm{U}$ test. $\mathrm{n}=3$, ${ }^{*} \mathrm{P}<0.05$. AR, androgen receptor; DU145 SCR, control cells; DU145 SH, zinc finger E-box binding homeobox 1-knockdown cells.

Statistical analysis. For statistical analysis of the data, the Mann-Whitney U test was applied using GraphPad Prism 5 software (GraphPad Software, Inc., La Jolla, CA, USA). Results were normalized to the results from DU145 SCR cells. The samples were processed in triplicate $(n=3)$ and data are expressed as the means \pm standard deviation. $\mathrm{P}<0.05$ was considered to indicate a statistically significant difference. 

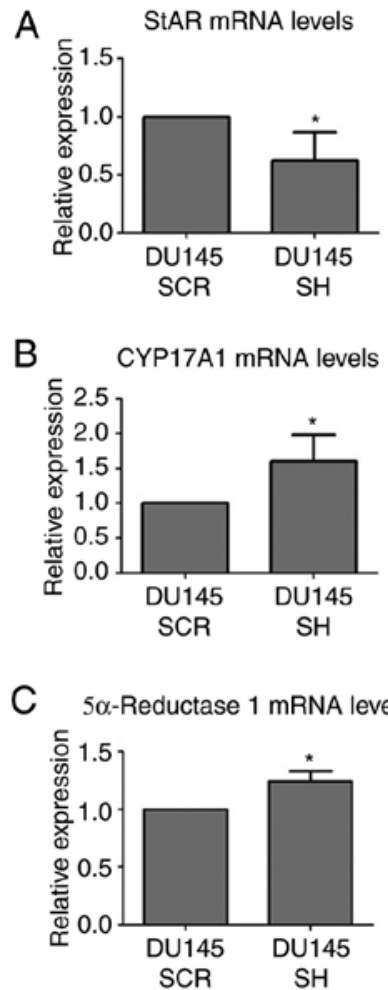

D $5 \alpha$-Reductase 2 mRNA levels

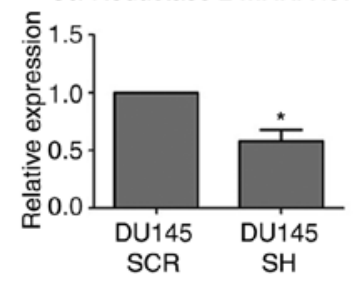

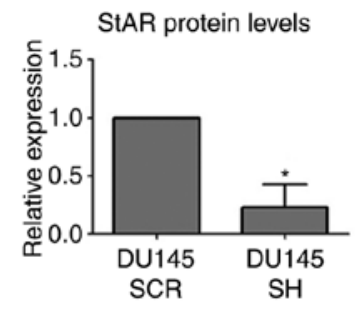
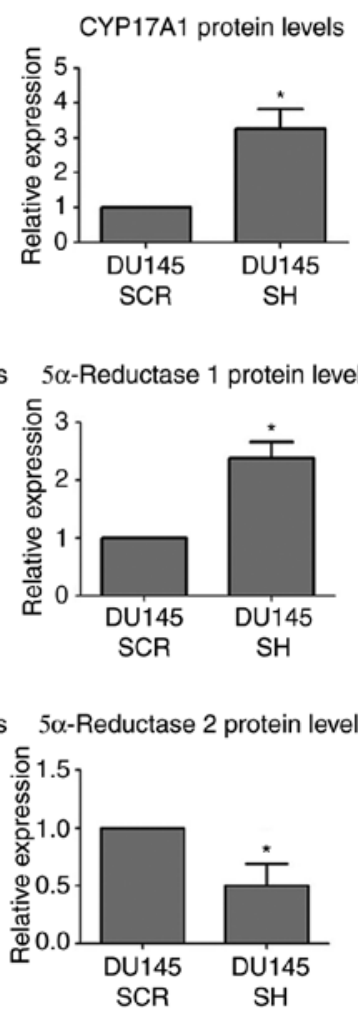
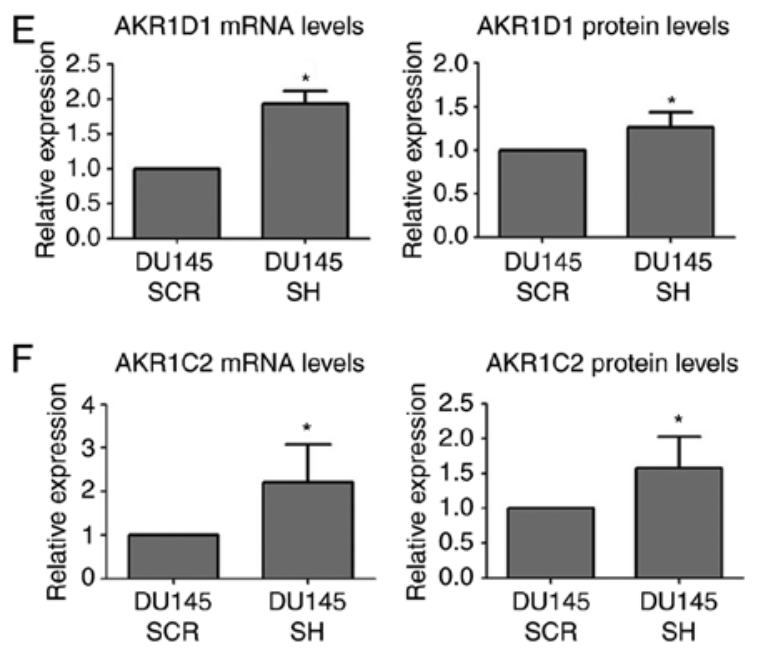

G

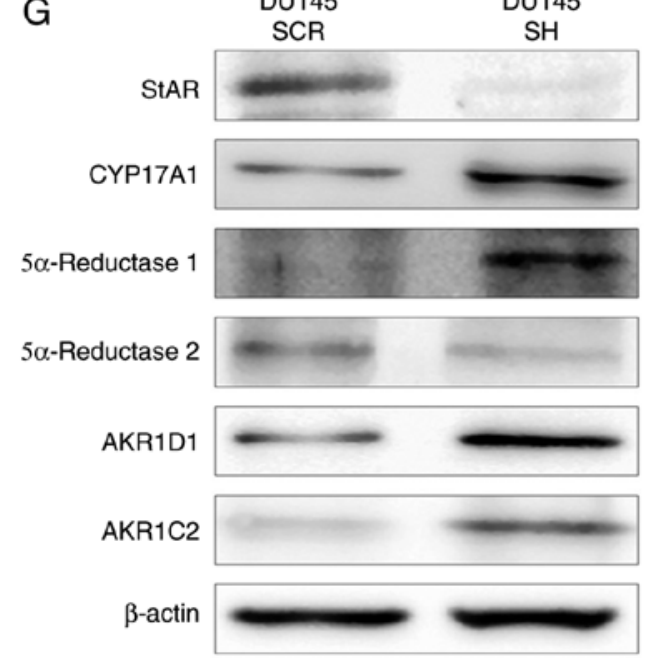

Figure 2. mRNA and protein expression levels of androgen synthesis and degradation markers in DU145 SCR and DU145 SH cell lines, as determined by reverse transcription-quantitative polymerase chain reaction and western blotting. mRNA and protein expression levels of (A) StAR, (B) CYP17A1, (C) $5 \alpha$-reductase-1, (D) $5 \alpha$-reductase-2, (E) AKR1D1 and (F) AKR1C2. (G) Western blot analysis; $\beta$-actin was used as a loading control. Statistically significant differences were determined using the Mann-Whitney U test. $n=3$, ${ }^{*} \mathrm{P}<0.05$. AKR1C2, aldo-keto reductase family 1 member $\mathrm{C} 2$; AKR1D1, aldo-keto reductase family 1 member D1; CYP17A1, cytochrome P450 family 17 subfamily A member; DU145 SCR, control cells; DU145 SH, zinc finger E-box binding homeobox 1-knockdown cells; StAR, steroidogenic acute regulatory protein.

\section{Results}

mRNA and protein expression, and cellular localization of the AR in a PCa cell line with ZEB1 silencing. Analysis of AR expression and subcellular localization demonstrated that ZEB1 silencing resulted in an increase in the mRNA and protein expression levels of AR (Fig. 1A and B), and AR was predominantly located in the perinuclear region (Fig. 1C).

$m R N A$ and protein expression, and cellular localization of

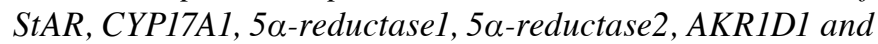
$A K R 1 C 2$ in a PCa cell line with ZEB1 silencing. The present study aimed to determine ZEB1 knockdown-induced alterations in the steroidogenic pathway (Figs. 2 and 3). Initially, the expression levels of the StAR transporter, which is involved in the first stage of the pathway $(11,12)$, were evaluated by RT-qPCR, western blotting and immunocytochemistry. The results obtained indicated that the mRNA expression levels of the StAR were decreased in the DU145 SH cell line compared with in control DU145 SCR cells. Furthermore, the protein expression levels of StAR were also reduced in DU145 SH cells (Fig. 2A and G). The intracellular distribution of StAR was mainly cytoplasmic (Fig. 3A).

The enzyme CYP17A1, which participates in the third and fourth step of the steroidogenic pathway $(11,12)$, was also analyzed by RT-qPCR, western blotting and immunocytochemistry. The results demonstrated that ZEB1 silencing resulted in an increase in the mRNA and protein expression levels of CYP17A1 compared with in the non-silenced control (Fig. 2B and G). In addition, the intracellular distribution exhibited a cytoplasmic pattern (Fig. 3B).

Within the enzymes involved in androgen synthesis, two isoforms were analyzed, $5 \alpha$-reductase 1 and $5 \alpha$-reductase 2 , which convert testosterone to DHT (12). The mRNA and protein expression levels of $5 \alpha$-reductase 1 were increased in the ZEB1-silenced cells (Fig. 2C and G). With regards to its subcellular localization, $5 \alpha$-reductase 1 was predominantly detected in the cytoplasm of DU145 SH and DU145 SCR 

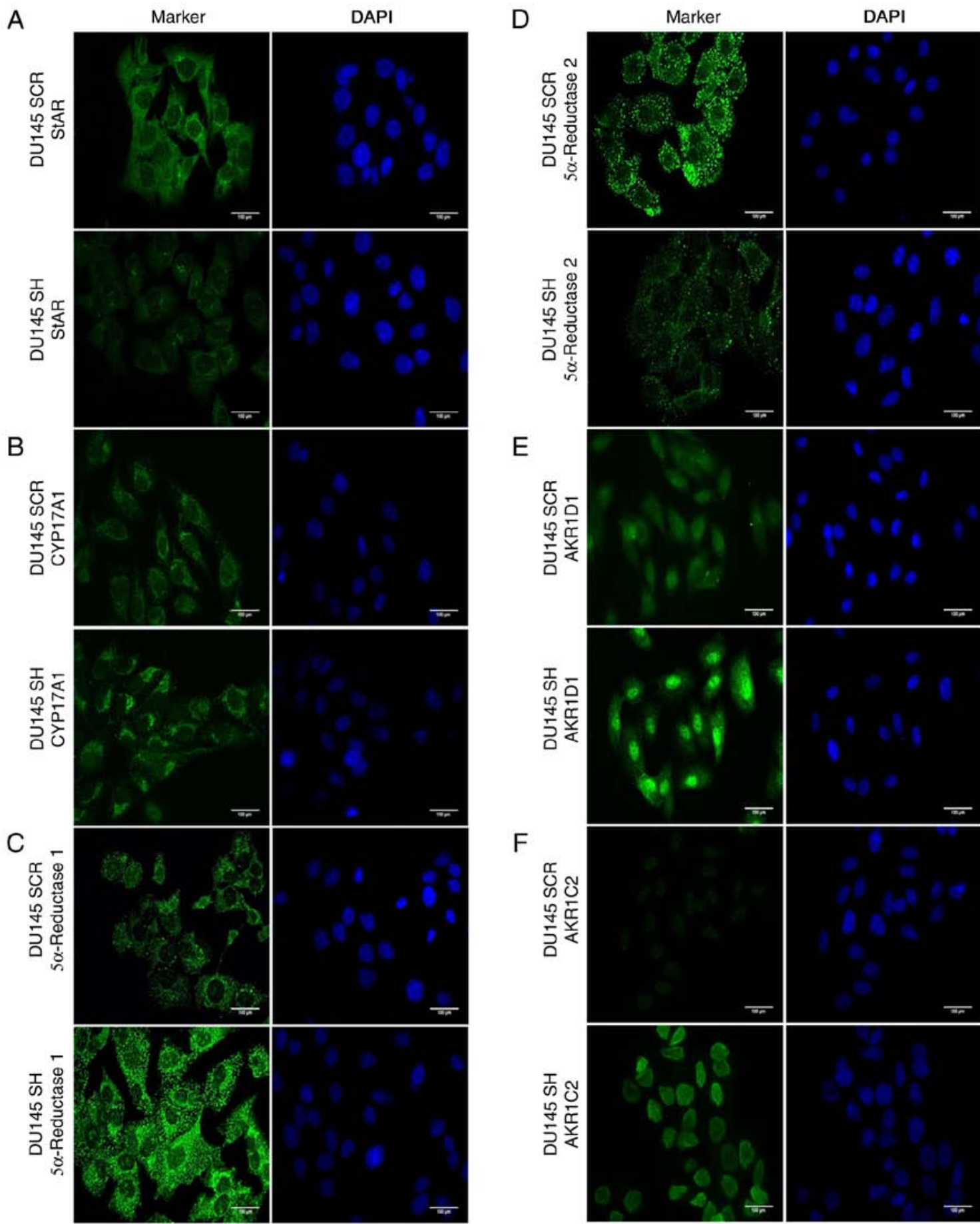

Figure 3. Immunocytochemistry of androgen synthesis and degradation markers in DU145 SH and DU145 SCR cell lines. Right panel, cell nuclei are shown in blue (DAPI); left panel, markers are shown in green (Alexa Fluor ${ }^{\circledR} 488$ ). (A) StAR, (B) CYP17A1, (C) $5 \alpha$-reductase-1, (D) $5 \alpha-$ reductase-2, (E) AKR1D1 and (F) AKR1C2. magnification, $\mathrm{x} 40 . \mathrm{n}=3$. AKR1C2, aldo-keto reductase family 1 member C2; AKR1D1, aldo-keto reductase family 1 member D1; CYP17A1, cytochrome P450 family 17 subfamily A member; DU145 SCR, control cells; DU145 SH, zinc finger E-box binding homeobox 1-knockdown cells; StAR, steroidogenic acute regulatory protein.

cells (Fig. 3C). Conversely, ZEB1 silencing induced a decrease in the mRNA and protein expression levels of $5 \alpha$-reductase 2 compared with the corresponding control group (Fig. 2D and G). Furthermore, cytoplasmic intracellular distribution was detected in both cell lines (Fig. 3D).

The enzyme AKR1D1, which is responsible for inactivation of progesterone, 17OH-progesterone, androstenedione and testosterone, thus converting the substrates into $5 \beta$-metabolites (13), was analyzed. The results indicated that knockdown of ZEB1 resulted in an increase in the mRNA and protein expression levels of AKR1D1 compared with in non-silenced control cells (Fig. 2E and G). In addition, the results of immunocytochemistry revealed that AKR1D1 exhibited nuclear location in DU145 SH cells (Fig. 3E).

Finally, the enzyme AKR1C2, which participates in the inactivation of DHT, was analyzed (14). The results revealed that ZEB1 silencing resulted in an increase in the mRNA and protein expression levels of AKR1C2 compared 
A

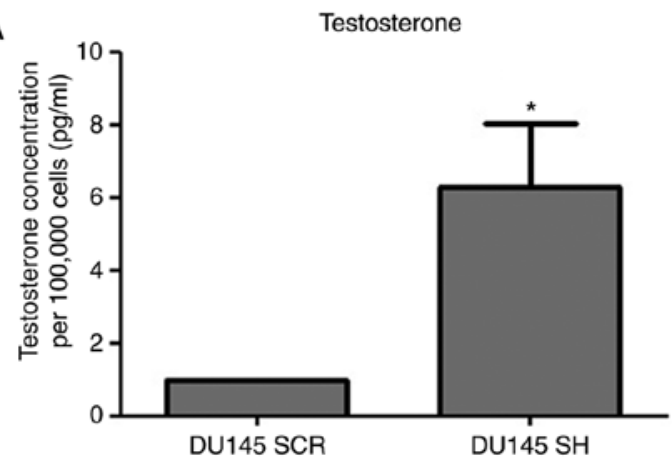

B

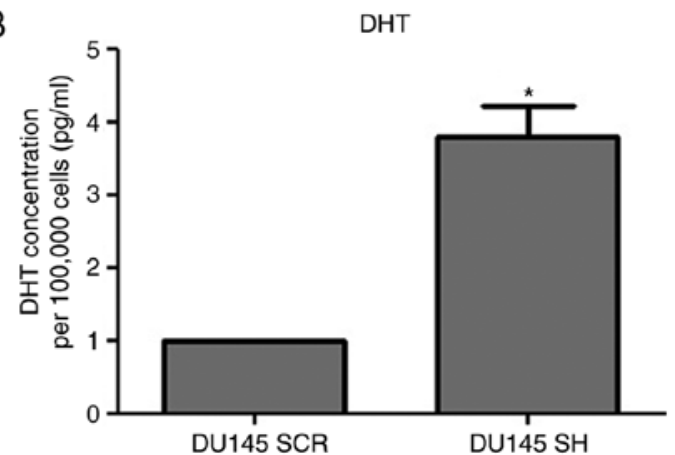

Figure 4. Testosterone and DHT concentration detected in the cell culture media of the DU145 SH and DU145 SCR cell lines. (A) Testosterone concentration $(\mathrm{pg} / \mathrm{ml}) / 10^{5}$ cells. (B) DHT concentration (pg/ml) $/ 10^{5}$ cells. Statistically significant differences were determined using the Mann-Whitney U test. $\mathrm{n}=3$, ${ }^{*} \mathrm{P}<0.05$. DHT, dihydrotestosterone DU145 SCR, control cells; DU145 SH, zinc finger E-box binding homeobox 1-knockdown cells.

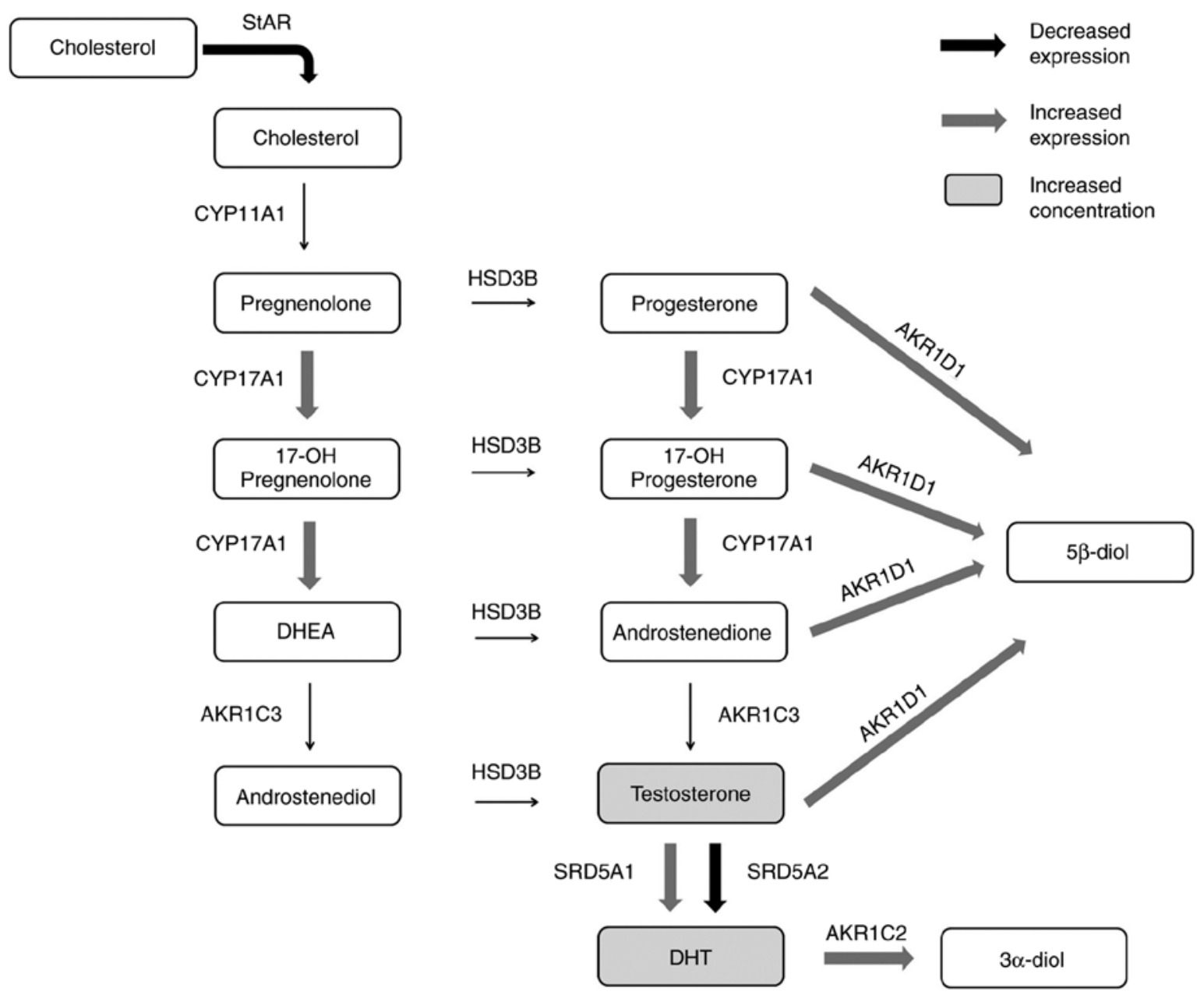

Figure 5. Summary of the results obtained regarding markers of the steroidogenic pathway in control DU145 cells and ZEB1-knockdown DU145 cells Androgen synthesis and degradation pathways are presented. Grey arrows indicate markers with increased expression in response to ZEB1 silencing; black arrows indicate markers with reduced expression in ZEB1-silenced cells; grey boxes indicate androgens with increased concentration in the cell culture media. ZEB1, zinc finger E-box binding homeobox 1 .

with in the corresponding controls (Fig. 2F and G). Furthermore, AKR1C2 was strictly located in the nuclei of DU145 SH cells and was weakly detected in DU145 SCR cells (Fig. 3F).

Testosterone and DHT concentrations in the culture medium of ZEB1-silenced PCa cells. The results revealed that ZEB1 silencing resulted in an increase in testosterone and DHT concentrations in DU145 SH cells compared with in the DU145 SCR control cells (Fig. 4).

A summary of the steroidogenic pathway markers, including those associated with androgen synthesis and degradation, in DU145 SH and DU145 SCR cell lines is presented in Fig. 5. 


\section{Discussion}

Our recent study revealed that DU145 cells express high levels of ZEB1; therefore, this cell line was selected to perform stable silencing in the present study. The results of our previous study demonstrated that cells with ZEB1 silencing undergo alterations in the expression levels of canonical markers of EMT, including E-cadherin and Vimentin; in particular, E-cadherin is increased and Vimentin is decreased in response to ZEB1 knockdown. In addition, it has been revealed that these cells exhibit a decrease in proliferation, migration and invasion (9). Taken together these results indicate that DU145 SH cells may acquire marked epithelial characteristics.

In the prostate, it has been widely described that testosterone synthesis begins with the transport of cholesterol to the inner mitochondrial membrane by StAR, followed by reactions catalyzed by the enzymes CYP11A1, CYP17A1, HSD3 $\beta$ and AKR1C3 (11). In the present study, some of these markers were analyzed, and the results suggested that an increase in CYP17A1 may contribute to an increase in testosterone synthesis in cells with ZEB1 silencing. Therefore, CYP17A1 may be considered the key enzyme in the production of testosterone in these cells. This finding is in accordance with the findings of Montgomery et al (15), which revealed that, in samples from patients with CRPC, an increase in the expression of CYP17A1 and testosterone concentration was detected compared with in primary prostate tumors samples, thus suggesting intratumoral steroidogenesis in advanced stages and progression to CRPC. The production of DHT may be determined by detecting the enzymes $5 \alpha$-reductase 1 and $5 \alpha$-reductase 2 , and testosterone concentration (16). The expression levels of the $5 \alpha$-reductase isoforms were detected in the present study and suggested that the main factor affecting DHT production may be the initial concentration of testosterone, since the decrease in one isoform was compensated with an increase in the other.

Testosterone and DHT concentrations are also determined by their inactivation and subsequent degradation $(13,14,16)$. Analysis of the enzyme AKR1D1, which is responsible for converting progesterone, $17 \mathrm{OH}$-progesterone, androstenedione and testosterone into inactive metabolites (13), revealed that its expression was increased in ZEB1-silenced cells, which may result in a greater inactivation of these hormone metabolites; however, the concentration of testosterone was increased in ZEB1-silenced cells. These findings suggested that the inactivation performed by AKR1D1 was not sufficient to prevent the high levels of testosterone in these cells. Similarly, the AKR1C2 enzyme, which is responsible for the inactivation of DHT, was also increased in cells with ZEB1 knockdown.

The preferential nuclear localization of AKR1D1 and AKR1C2 suggested that after being translocated from the cytoplasm, androgen receptor-testosterone and androgen receptor-DHT complexes (17) may be inactivated in the nucleus . Taylor et al (18) analyzed the genomic profile of samples from patients with $\mathrm{PCa}$, and revealed that AKR1C2 expression is increased in patients with advanced disease, thus suggesting that the increase in AKR1C2 expression determined in this study may be associated with more advanced stages of PCa. In addition, the expression levels of the enzyme AKR1C2 are correlated with the expression of AR (19). Huang et al (20) revealed that $\mathrm{LNCaP}$ PCa cells, which express AR, have a higher expression of AKR1C2 compared with in cell lines that do not express AR. It was also demonstrated that silencing AR in $\mathrm{LNCaP}$ cells produces a decrease in AKR1C2; however, the regulatory mechanism remains unknown. Furthermore, Ji et al (21) reported that DHT treatment of LNCaP and DU145 cells results in induction of AKR1C2 expression, indicating that this possibly occurs to counteract the increase in DHT concentration. These findings are in accordance with the present results, suggesting a possible regulation of androgen availability by the AR as a mechanism of control.

Notably, the relevance of increased concentration of androgens in cells with ZEB1 knockdown may be associated with activation of AR during EMT (6). Therefore, an increase in the expression of steroidogenic enzymes has been suggested as a mechanism underlying resistance to ADT $(22,23)$. According to the present results, it may be hypothesized that an increase in androgen synthesis could lead to an increase in the activation of AR due to its increased availability.

The present results revealed that the isoform $5 \alpha$-reductase 1 was increased whereas $5 \alpha$-reductase 2 was decreased in response to ZEB1 knockdown. An expression switch between these isoforms has been suggested, since both enzymes act at different $\mathrm{pH}$ levels, and in the tumor environment, the activity of $5 \alpha$-reductase 1 may be favored allowing an increase in the production of DHT (12). In addition Audet-Walsh et al (24) revealed that theinverseregulation of these enzymes is conducted directly by the AR, which binds to the promoter sequences of the genes increasing the expression of $5 \alpha$-reductase 1 and suppressing the expression of $5 \alpha$-reductase 2.

Locke et al (25) reported an increase in StAR expression, and testosterone and DHT concentrations, in a castration-resistant xenotransplant model. Conversely, the present results suggested that an inverse relationship may exist between the concentration of androgens and StAR. It may be hypothesized that a reduction in StAR could act as a compensatory mechanism against the increase in androgens induced by ZEB1-independent mechanisms.

In conclusion, the silencing of transcription factor ZEB1 produced an alteration in the steroidogenic pathway and AR expression, specifically increasing the expression levels of CYP17A1, 5 $\alpha$-reductase 1, AKR1D1 and AKR1C2 enzymes, and decreasing the expression levels of StAR and $5 \alpha$-reductase 2 . In addition, ZEB1 knockdown increased testosterone and DHT concentrations. An increase in androgen concentration in tumor cells is a characteristic of patients with ADT resistance; therefore, alterations in ZEB1 expression may be associated with the mechanism underlying treatment failure in patients undergoing ADT and subsequent disease progression to CRPC.

\section{Acknowledgements}

The authors would like to thank Ms. Graciela Caroca (Department of Basic and Clinic Oncology, Faculty of Medicine, University of Chile) for her technical assistance.

\section{Funding}

The present study was supported by grants from the Fondo Nacional de Ciencia y Tecnología, FONDECYT [grant 
nos. 1151214 (HRC) and 1140417 (EAC)]. U-REDES, University of Chile [grant no. URC-007/17 (HRC)], and the CONICYT scholarship [grant no. 21140772 (OOS) and 21160703 (MJT)].

\section{Availability of data and materials}

The datasets used and/or analyzed during the present study are available from the corresponding author on reasonable request.

\section{Authors' contributions}

DH performed the gene expression studies, immunocytochemistry, ELISA and the statistical analyses. OOS transduced the cell line, and contributed to the gene expression and immunocytochemistry experiments. PV and MJT contributed to the gene expression studies and protein analyses. HRC, RP and EAC participated in the study design and interpreted the results. All authors read and approved the final manuscript.

\section{Ethics approval and consent to participate}

Not applicable.

\section{Patient consent for publication}

Not applicable.

\section{Competing interests}

The authors declare that they have no competing interests.

\section{References}

1. Bray F, Ferlay J, Soerjomataram I, Siegel RL, Torre LA and Jemal A: Global cancer statistics 2018: GLOBOCAN estimates of incidence and mortality worldwide for 36 cancers in 185 countries. CA Cancer J Clin, 2018.

2. Anantharaman A and Frieddlaner TW: Targeting the androgen receptor in metastatic castrate-resistant prostate cancer: A review. Urol Oncol 34: 356-367, 2016.

3. Lonergan PE and Tindall DJ: Androgen receptor signaling in prostate cancer development and progression. J Carcinog 10: 20, 2011.

4. Chandrasekar T, Yang JC, Gao AC and Evans CP: Mechanisms of resistance in castration-resistant prostate cancer (CRPC). Transl Androl Urol 4: 365-380, 2015.

5. Mitsiades N, Sung C, Schultz N, Danila DC, He B, Eedunuri VK, Fleisher M, Sander C, Sawyers CL and Scher HI: Distinct patterns of dysregulated expression of enzymes involved in androgen synthesis and metabolism in metastatic prostate cancer tumors. Cancer Res 72: 6142-6152, 2012.

6. Banerjee P, Banerjee S, Brown T and Zirkin B: Androgen action in prostate function and disease. Am J Clin Exp Urol 6: 62-77, 2018.

7. Sun Y, Wang BE, Leong KG, Yue P, Li L, Jhunjhunwala S, Chen D, Seo K, Modrusan Z, Gao WQ, et al: Androgen deprivation causes epithelial-mesenchymal transition in the prostate: Implications for androgen-deprivation therapy. Cancer Res 72 $527-536,2012$
8. Anose BM and Sanders MM: Androgen receptor regulates transcription of the ZEB1 transcription factor. Int J Endocrinol 2011: 903918, 2011.

9. Orellana-Serradell O, Herrera D, Castellon E and Contreras HR: The transcription factor ZEB1 promotes an aggressive phenotype in prostate cancer cell lines. Asian J Androl 20: 294-299, 2018.

10. Livak KJ and Schmittgen TD: Analysis of relative gene expression data using real-time quantitative PCR and the $2^{-\Delta \Delta C_{\mathrm{T}}}$ method. Methods 25: 402-408, 2001.

11. Payne AH and Hales DB: Overview of steroidogenic enzymes in the pathway from cholesterol to active steroid hormones. Endocr Rev 25: 947-970, 2004.

12. Mostaghel EA: Steroid hormone synthetic pathways in prostate cancer. Transl Androl Urol 2: 212-227, 2013.

13. Chen $M$ and Penning TM: $5 \beta$-Reduced steroids and human $\Delta^{4}$-3-ketosteroid 5 $\beta$-reductase (AKR1D1). Steroids 83: 17-26, 2014.

14. Zeng CM, Chang LL, Ying MD, Cao J, He QJ, Zhu H and Yang B: Aldo-keto reductase AKR1C1-AKR1C4: Functions, regulation, and intervention for anti-cancer therapy. Front Pharmacol 8: 119, 2017.

15. Montgomery RB, Mostaghel EA, Vessella R, Hess DL, Kalhorn TF, Higano CS, True LD and Nelson PS: Maintenance of intratumoral androgens in metastatic prostate cancer: A mechanism for castration-resistant tumor growth. Cancer Res 68 : 4447-4454, 2008.

16. Rizner TL, Lin HK, Peehl DM, Steckelbroeck S, Bauman DR and Penning TM: Human type 3 3alpha-hydroxysteroid dehydrogenase (aldo-keto reductase $1 \mathrm{C} 2$ ) and androgen metabolism in prostate cells. Endocrinology 144: 2922-2932, 2003.

17. Basu S and Tindall DJ: Androgen action in prostate cancer. Horm Cancer 1: 223-228, 2010.

18. Taylor BS, Schultz N, Hieronymus H, Gopalan A, Xiao Y, Carver BS, Arora VK, Kaushik P, Cerami E, Reva B, et al: Integrative genomic profiling of human prostate cancer. Cancer Cell 18: 11-22, 2010.

19. Zhang A, Zhang J, Plymate S and Mostaghel EA: Classical and non-classical roles for pre-receptor control of DHT metabolism in prostate cancer progression. Horm Cancer 7: 104-113, 2016.

20. Huang KH, Chiou SH, Chow KC, Lin TY, Chang HW, Chiang IP and Lee MC: Overexpression of aldo-keto reductase 1C2 is associated with disease progression in patients with prostatic cancer. Histopathology 57: 384-394, 2010.

21. Ji Q, Chang L, VanDenBerg D, Stanczyk FZ and Stolz A: Selective reduction of AKR1C2 in prostate cancer and its role in DHT metabolism. Prostate 54: 275-289, 2003.

22. Kobayashi T, Inoue T, Kamba T and Ogawa O: Experimental evidence of persistent androgen-receptor-dependency in castration-resistant prostate cancer. Int J Mol Sci 14: 15615-15635, 2013.

23. Lubik A, Nouri M, Truong S, Ghaffari M, Adomat HH, Corey E, Cox ME, Li N, Guns ES, Yenki P, et al: Paracrine sonic hedgehog signaling contributes significantly to acquired steroidogenesis in the prostate tumor microenvironment. Int J Cancer 140: 358-369, 2017.

24. Audet-Walsh É, Yee T, Tam IS and Giguère V: Inverse regulation of DHT synthesis enzymes $5 \alpha$-reductase types 1 and 2 by the androgen receptor in prostate cancer. Endocrinology 158: 1015-1021, 2017

25. Locke JA, Guns ES, Lubik AA, Adomat HH, Hendy SC, Wood CA, Ettinger SL, Gleave ME and Nelson CC: Androgen levels increase by intratumoral de novo steroidogenesis during progression of castration-resistant prostate cancer. Cancer Res 68: 6407-6415, 2008 